\title{
Enfermedad COVID-19 en adultos jóvenes mexicanos hospitalizados
}

\section{COVID-19 disease in hospitalized Mexican young adults}

\author{
Carla Paola Sánchez-Ríos, ${ }^{*}$ Oscar Gabriel Jiménez-Cabrera, ${ }^{\ddagger}$ \\ Omar Barreto-Rodríguez, ${ }^{*}$ Norma Angélica Téllez-Navarrete* \\ *Instituto Nacional de Enfermedades Respiratorias Ismael Cosío Villegas, Ciudad de México. \\ ${ }^{\ddagger}$ Instituto de Seguridad Social del Estado de México y Municipios.
}

\begin{abstract}
RESUMEN. Introducción: La literatura sugiere que el comportamiento de la infección por SARS-CoV-2 en jóvenes es diferente. Se ha reportado que COVID-19 es menos frecuente y menos grave; sin embargo, la evidencia sigue siendo contrastante. Este estudio tuvo el objetivo de describir la enfermedad COVID-19 en jóvenes mexicanos hospitalizados. Material y métodos: Se realizó un estudio retrospectivo en 102 pacientes con COVID-19 ingresados al Instituto Nacional de Enfermedades Respiratorias Ismael Cosío Villegas y Centro Médico del Instituto de Seguridad Social del Estado de México y Municipios (ISSEMyM) Toluca, Estado de México, en el período de marzo a mayo del 2020. Se clasificaron de acuerdo con la edad, 51 pacientes en el grupo de adultos jóvenes $<35$ años, y 51 adultos $>35$ años. Se compararon las características clínicas entre los grupos y su asociación con desenlace de la gravedad de enfermedad y muerte. Resultados: En el grupo de jóvenes (<35 años) la saturación de $\mathrm{O}_{2}$ al ingreso fue de 86\% IQR (74-91), 66\% $(\mathrm{n}=34)$ presentó linfopenia al ingreso. El porcentaje de obesidad en jóvenes fue similar al de los adultos (43 versus $41 \%$ ). El grado de severidad fue leve en $27.5 \%(n=14)$, moderada en $45 \%(n=23)$ y grave/crítica en $27.5 \%(n=14)$. Este grupo presentó menores grados de taquipnea, gravedad de COVID-19 y letalidad por infección por SARS-CoV-2 al ser comparados con adultos (15 versus $45 \%, \mathrm{p}<0.05$ ). $\mathrm{Al}$ ingreso hospitalario los valores de dímero $\mathrm{D}$ fueron menores en este grupo ( 0.69 versus $1.01 \mu \mathrm{g} / \mathrm{mL})$ y la albúmina sérica fue mayor ( 3.77 versus $3.3 \mathrm{mg} / \mathrm{dL}$ ) comparado con los valores en adultos. La relación neutrófilo/linfocito y plaquetas/linfocitos se encontraron como posibles biomarcadores predictores de deterioro por COVID-19 con un OR de 8.03 y 3.6, respectivamente. Conclusión: Los adultos jóvenes con COVID-19 que ameritan hospitalización tienen un alto porcentaje de obesidad. Tienen mayor cantidad de albumina inicial y niveles más bajos de dímero $\mathrm{D}$ con cuadros frecuentemente leves-moderados al ingreso, y menos letales comparados con los enfermos adultos hospitalizados.
\end{abstract}

Palabras clave: COVID-19, jóvenes, biomarcador, severidad.

Correspondencia: Dra. Carla Paola Sánchez-Ríos

Instituto Nacional de Enfermedades Respiratorias

Ismael Cosío Villegas, Ciudad de México.

Correo electrónico: pao1144tosto@gmail.com

Recibido: 14-I-2021; aceptado: 07-IV-2021.

Citar como: Sánchez-Ríos CP, Jiménez-Cabrera OG, Barreto-Rodríguez O, Téllez-Navarrete NA. Enfermedad COVID-19 en adultos jóvenes mexicanos hospitalizados. Neumol Cir Torax. 2021; 80 (2): 105-110. https://dx.doi. org/10.35366/100991
ABSTRACT. Introduction: The literature suggests that the behavior of SARS-CoV-2 infection in young people is different. COVID-19 has been reported to be less frequent and less severe yet the evidence remains contrasting. This study had the objective of describing the COVID-19 disease in hospitalized Mexican youth. Material and methods: A retrospective study was carried out in 102 patients with COVID-19 admitted to the National Institute of Respiratory Diseases Ismael Cosío Villegas and the ISSEMyM Toluca State of Mexico Medical Center in the period from March to May 2020. They were classified according to age, 51 patients in the group of young adults $<35$ years and 51 adults older than 35 years. The clinical characteristics between the groups and their association with decreased severity of illness and death were compared. Results: In the group of young people $\left(<35\right.$ years) the saturation of $\mathrm{O}_{2}$ at admission was $86 \%$ IQR $(74-91) .66 \%(n=34)$ presented lymphopenia on admission. The percentage of obesity in young people was similar to that of adults ( $43 \%$ versus $41 \%$ ). The degree of severity was mild in $27.5 \%(\mathrm{n}=14)$, moderate in $45 \%(\mathrm{n}=23)$ and severe/critical in $27.5 \%$ $(n=14)$. This group also presented lower degrees of tachypnea, severity of COVID-19 and lethality due to SARS-CoV-2 infection when compared to adults $(15 \%$ versus $45 \% \mathrm{p}<0.05)$. At hospital admission, $\mathrm{D}$-dimer values were lower in this group $(0.69$ versus $1.01 \mu \mathrm{g} / \mathrm{mL})$ and serum albumin was higher ( 3.77 versus $3.3 \mathrm{mg} / \mathrm{dL}$ ) compared with the values in adults. The neutrophil/lymphocyte ratio and platelets/lymphocytes were found as possible predictive biomarkers of deterioration by COVID-19 with an OR of 8.03 and 3.6, respectively. Conclusion: Young adults with COVID-19 who warrant hospitalization have a high percentage of obesity. They have a higher amount of initial albumin, and lower levels of D-dimer with symptoms that are frequently mild-moderate on admission and less lethal compared to hospitalized adult patients.

Keywords: COVID-19, young people, biomarker, severity.

\section{INTRODUCCIÓN}

La nueva enfermedad por coronavirus (COVID-19) causada por el SARS-CoV-2 desde su inicio se ha propagado rápidamente. ${ }^{1,2}$ Los coronavirus representan un grupo heterogéneo de grandes virus de ARN de cadena sencilla, ampliamente distribuidos entre mamíferos y aves, agrupados en la familia de Coronaviridae. ${ }^{3}$ Los géneros de interés para los humanos son los coronavirus alfa y beta que componen la subfamilia 
de Coronaviridae, junto con los coronavirus gamma y delta, no reconocidos como patógenos humanos. ${ }^{4} \mathrm{~A}$ finales de la segunda década del siglo XXI, el mundo ha sido testigo de un brote de un nuevo coronavirus designado SARS-CoV-2, y asignado taxonómicamente a las especies de coronavirus agudo severo relacionado con el síndrome respiratorio agudo (SARS-CoV), subgénero sarbecovirus, género betacoronavirus. ${ }^{5,6}$ El término acuñado para referirse a la enfermedad relacionada con el SARS-CoV-2 es COVID-19.' Las gotas y el contacto cercano se han reconocido como las principales vías de transmisión del virus SARS-CoV-2 en COVID-19; aunque la ruta fecal-oral no puede excluirse. ${ }^{9,10}$

El escenario clínico de COVID-19 es heterogéneo. De hecho, el espectro de la enfermedad varía desde síntomas leves como fiebre, tos seca y disnea, hasta formas moderadas con síndrome de dificultad respiratoria aguda (SDRA); o bien formas, graves y críticas donde se presenta SDRA con necesidad de ventilación mecánica, o sepsis y falla orgánica múltiple secundaria. ${ }^{11-13}$ Además, también se ha informado de un curso asintomático, lo que hace que la contención de la infección sea más difícil. Los niños y jóvenes tienden a presentar enfermedad COVID-19 clínica con menor frecuencia y con cuadros relativamente menos graves; sin embargo, los estudios de seroprevalencia reportan tasas de infección similar a otros grupos de edad.

Por otro lado, entre los pacientes infectados con SARS$\mathrm{CoV}-2$, varias afecciones están relacionadas con una mayor susceptibilidad al virus y una carga elevada de COVID-19, lo que impacta en la gravedad del curso de la enfermedad.

Al día de hoy, la evidencia sugiere que la edad avanzada y las comorbilidades preexistentes son los predictores más importantes para el desenlace fatal. ${ }^{14}$ Empero, también se ha mencionado que los pacientes jóvenes cuentan con un sistema inmunológico apto para montar respuestas y cascadas inflamatorias potentes que pueden generar en algunos escenarios respuestas exageradas y, en consecuencia, complicaciones secundarias a la infección viral. ${ }^{15}$ En la población mexicana no se ha estudiado el comportamiento de la enfermedad COVID-19 en adultos jóvenes, por lo que se realizó el presente estudio de investigación con el objetivo de comparar las características clínicas en esta población, la presentación de la enfermedad y su desenlace clínico.

\section{MATERIAL Y MÉTODOS}

Población de estudio. Se realizó un estudio retrospectivo, donde se evaluaron 102 pacientes ingresados con diagnóstico de COVID-19 durante el período de marzo a mayo del 2020 en dos centros de referencia; 91 pacientes procedentes del Instituto Nacional de Enfermedades Respiratorias Ismael Cosío Villegas (INER) localizado en el sur de la Ciudad de México, y 11 pacientes del Centro Médico del Instituto de Seguridad Social del Estado de México y Municipios (ISSEMyM), localizado en el municipio de Toluca del Estado de México. Se incluyeron sujetos mayores de 18 años que ingresaron con diagnóstico de COVID-19. Se incluyeron los pacientes que contaran con expediente completo con datos clínicos, estudios de laboratorio y de imagen para fines del estudio. Se agruparon los pacientes para su análisis por rango de edad en dos grupos de estudio: adulto joven y adulto.

Definiciones de grupos y enfermedad. Debido a las diversas definiciones en cuanto a los límites de edad y clasificación de la enfermedad, se decidió con base en las definiciones por rangos etarios por organizaciones de salud y al criterio del investigador las siguientes definiciones.

La escala de evaluación de afectación tomográfica en COVID-19 propuesta por el Servicio de Radiología e Imagen del Instituto Nacional de Enfermedades Respiratorias Ismael Cosío Villegas: valoración semicuantitativa de extensión de lesiones por lóbulos pulmonares:

$\begin{array}{cc}\text { Puntuación } & \text { Rangos }(\%) \\ 1 & \leq 5 \\ 2 & >5-25 \\ 3 & >25-50 \\ 4 & >50-75 \\ 5 & >75\end{array}$

$\begin{array}{lc}\text { Interpretación } & \\ \text { Afección leve } & 1-5 \text { ptos } \\ \text { Afección moderada } & >5-15 \text { ptos } \\ \text { Afección severa } & >15 \text { ptos }\end{array}$

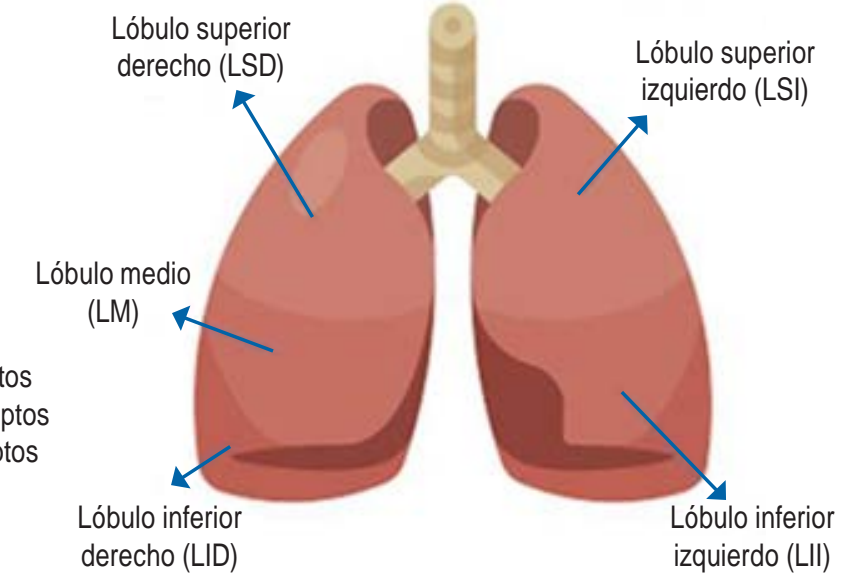

Figura 1:

Cuantificación de afección tomográfica por enfermedad COVID-19 propuesta por el Instituto Nacional de Enfermedades Respiratorias Ismael Cosío Villegas (INER). 
Tabla 1: Características basales clínicas y demográficas de la población de estudio.

\begin{tabular}{|c|c|c|c|}
\hline Características & Adultos jóvenes ( $\mathrm{N}=51$ ) & Adultos $\geq 35$ años $(\mathrm{N}=51)$ & $\mathrm{p}^{*}$ \\
\hline Edad & $30(27-33)$ & $47(41-52)$ & $<0.05$ \\
\hline Hombres, n (\%) & $37(72.0)$ & $40(78.0)$ & ns \\
\hline IMC $\left(\mathrm{kg} / \mathrm{m}^{2}\right)$ & $29(25-34)$ & $28(26-31)$ & ns \\
\hline Sobrepeso $\geq 25\left(\mathrm{~kg} / \mathrm{m}^{2}\right), \mathrm{n}(\%)$ & $18(35.0)$ & $27(52.0)$ & ns \\
\hline Obesidad $\geq 30\left(\mathrm{~kg} / \mathrm{m}^{2}\right), \mathrm{n}(\%)$ & $22(43.0)$ & $21(41.0)$ & ns \\
\hline Diabetes, $n(\%)$ & $4(7.8)$ & $9(17.6)$ & ns \\
\hline HAS, $n(\%)$ & $2(3.9)$ & $5(9.8)$ & ns \\
\hline Tabaquismo, n (\%) & $14(27.0)$ & $16(31.0)$ & ns \\
\hline Saturación $\mathrm{O}_{2}(\%)$ ingreso & $86(74-91)$ & $79(63-86)$ & $<0.05$ \\
\hline Neutrófilos $\times 1,000 \mathrm{cel} / \mathrm{mm}^{3}$ & $6.0(4-9.5)$ & $7.8(5-10)$ & ns \\
\hline Linfocitos $\times 1,000 \mathrm{cel} / \mathrm{mm}^{3}$ & $0.9(0.7-1.4)$ & $0.8(0.6-1.1)$ & ns \\
\hline Linfopenia < $1.1 \mathrm{cel} / \mathrm{mm}^{3}, \mathrm{n}(\%)$ & $34(66.0)$ & $39(76.0)$ & ns \\
\hline Relación neutrófilos/linfocitos & $6.1(3.8-10.9)$ & $9(4.6-16)$ & 0.052 \\
\hline Relación plaquetas/infocitos & $229(164-374)$ & $260(190-380)$ & ns \\
\hline Hemoglobina (mg/dL) & $14.7(13-15)$ & $15.4(14.7-16.4)$ & $<0.05$ \\
\hline DHL (UI/L) & 398 (266-498) & $383(319-614)$ & ns \\
\hline Albúmina (mg/dL) & $3.77(3.6-4.0)$ & $3.3(3.0-3.7)$ & $<0.05$ \\
\hline Dímero D $(\mu \mathrm{g} / \mathrm{mL})$ & $0.69(0.37-1.0)$ & $1.01(0.66-3.05)$ & $<0.05$ \\
\hline
\end{tabular}

${ }^{*}$ Análisis estadístico U-Mann Whitney $/ \chi^{2} . p<0.05$ a dos colas.

$I M C=$ índice de masa corporal; $H A S=$ hipertensión arterial; $D H L=$ deshidrogenasa láctica; ns = no significativo.

Datos expresados con mediana e intervalo intercuartilar (IQR) 25-75 y n con porcentajes.

Adulto joven: persona que se encuentra en rango de edad de 17 a 34 años. Adulto: persona que se encuentra en rango de edad de > 35-60 años.

Definiciones de la COVID-19 de acuerdo con la OMS: Enfermedad leve: paciente sintomático que se ajusta a la definición de caso de COVID-19, pero no presenta neumonía vírica ni hipoxia. Enfermedad moderada: neumonía con signos clínicos de neumonía (fiebre, tos, disnea, taquipnea), pero sin signos de neumonía grave, en particular $\mathrm{SpO}_{2} \geq 90 \%$ con aire ambiente. Enfermedad grave/crítica: neumonía grave con signos clínicos de neumonía (fiebre, tos, disnea, taquipnea) más alguno de los siguientes: frecuencia respiratoria $>30$ inspiraciones/min, dificultad respiratoria grave o $\mathrm{SpO}_{2}<90 \%$ al aire ambiente. Se incluye en este grupo a los casos que desarrollaron también síndrome de dificultad respiratoria aguda (SDRA). Definición de severidad de neumonía: con base en el esquema planteado por el Servicio de Radiología Torácica del INER Ismael Cosío Villegas, Ciudad de México (Figura 1).

Análisis estadístico. En el análisis descriptivo, las variables cualitativas se expresaron como porcentajes, las variables continuas se expresaron como mediana e intervalo intercuartilar (IQR 25-75). Las diferencias entre los dos grupos se compararon con las pruebas $U$ Mann-Whitney o $\chi^{2}$ según fuera el caso. Para la asociación de los grupos para desenlace de muerte se realizó análisis univariado. Se consideró significativo un valor de $p<0.05$ a dos colas. Los datos se analizaron en el software Stata/SE V.13.0.

\section{RESULTADOS}

Se analizó una muestra total de 102 pacientes, 51 jóvenes y 51 adultos jóvenes mayores de 35 años con diagnóstico de enfermedad COVID-19 confirmada por RT-PCR de exudado nasofaríngeo o con clínica e imagen compatible con enfermedad en el período de estudio. Las variables clínicas entre los dos grupos se muestran en la Tabla 1. La mediana de edad entre los grupos fue de 30 IQ (27-33) años versus 47 IQ (41-52) años. En el grupo de jóvenes 72\% $(n=37)$ fueron hombres. Respecto a las comorbilidades presentadas por los pacientes menores de 35 años, destaca asma en $3.9 \%(n=2)$; sobrepeso $35 \%(n=18)$; obesidad $43 \%$ $(n=22)$; diabetes en $7.8 \%(n=4)$; hipertensión arterial sistémica en $3.9 \%(n=2)$. No se reportó cáncer o 
enfermedad renal/hepática crónica. El tabaquismo activo fue reportado en $27 \%(n=14)$, sin diferencia significativa en las comorbilidades con respecto a los mayores de 35 años.

En el grupo de adultos jóvenes la prueba de RT-PCR para SARS-CoV-2 de exudado nasofaríngeo inicial se reportó positiva en $90.6 \%(n=46)$ de pacientes. La mediana de frecuencia respiratoria al ingreso fue de 22 IQR (20-30), saturación por oximetría de pulso inicial fue de $86 \%$ IQR (74-91) al aire ambiente. El grado de severidad de la enfermedad COVID-19 al momento del ingreso hospitalario en este grupo fue: leve en $27.5 \%(n=14)$, moderada en $45 \%$ $(n=23)$ y grave/crítica en $27.5 \%(n=14) ; 76 \%(n=39)$ consultó atención médica después de presentar síntomas por más de cinco días (Tabla 2).

En cuanto a las variables de laboratorio clínico, observamos que $66 \%(n=34)$ presentó linfopenia al ingreso. La mediana de la relación neutrófilo/linfocito fue de 6.16 IQR (3.8-0.9) y la de plaquetas/linfocito fue de 229 IQR (164-374), la mediana de DHL fue de 398 IQR (266-498), albúmina 3.77 IQR (3.6-4.0) y dímero D 0.69 IQR (0.37-1.0).

El dímero $\mathrm{D}$ se encontró menor (0.69 versus $1.01 \mu \mathrm{g} /$ $\mathrm{mL}$ ) y la albúmina sérica mayor (3.77 versus $3.3 \mathrm{mg} / \mathrm{dL}$ ) al ingreso en jóvenes hospitalizados ( $p<0.05)$ (Figura 2).

Respecto a la mortalidad, en el grupo de jóvenes por COVID-19 fue de $15 \%(n=8)$ con diferencia significativa contra el grupo de adultos de más de 35 años $(p<0.05)$ (Tabla 3).

\section{DISCUSIÓN}

El comportamiento de la enfermedad COVID-19 es distinto en los diferentes grupos etarios y esto ha quedado demostrado en la literatura mundial. Swann et al. encontraron que en las personas jóvenes los síntomas de presentación más comunes de esta enfermedad son fiebre, tos, náusea, vómito y disnea. ${ }^{16}$ Hoy en día también se ha demostrado que la severidad de síntomas puede influir en la búsqueda de atención médica por parte de los enfermos. Los niños y adultos jóvenes tienden a presentar cuadros leves y esto puede incluso contribuir a las falsas estadísticas de pacientes COVID-19 por no buscar atención médica y realización
Tabla 2: Clasificación clínica de COVID-19 de acuerdo con la edad.

\begin{tabular}{|l|c|c|c|}
\hline & $\begin{array}{c}\text { Adultos jóvenes } \\
(\mathbf{N}=51)\end{array}$ & $\begin{array}{c}\text { Adultos } \geq 35 \\
\text { años (N=51) }\end{array}$ & \\
\hline $\begin{array}{l}\text { Forma clínica } \\
\text { de COVID-19 }\end{array}$ & $\mathbf{n}(\%)$ & $\mathbf{n}(\%)$ & $\mathbf{p}^{*}$ \\
\hline Leve & $14(27.5)$ & $5(10.0)$ & $<0.05$ \\
Moderada & $23(45.0)$ & $19(37.0)$ & $<0.05$ \\
Grave & $14(27.5)$ & $27(53.0)$ & $<0.05$ \\
Muerte & $8(15.0)$ & $23(45.0)$ & $<0.05$ \\
\hline
\end{tabular}

${ }^{*}$ Prueba estadística $\chi^{2}$.

Datos expresados con $n$ y porcentajes.

de pruebas microbiológicas, incluso manteniéndose así durante todo el cuadro de infección. Los cuadros leves iniciales en las personas jóvenes favorecen que, en los pacientes en los que el cuadro evoluciona tórpidamente, cuando buscan atención médica la temporalidad de la enfermedad se encuentra en fase más avanzada y esto impacta en el pronóstico. En nuestro estudio se encontró que en promedio las personas menores de 35 años buscaron atención médica en su mayoría después de cinco días de haber iniciado el cuadro. Las comorbilidades más frecuentes documentadas en infectados por SARS-CoV-2 dependen de la edad, del tipo de centro hospitalario e inclusive de la región. Si bien, las más reportadas en niños son comorbilidades neurológicas seguidas de las hematológico/oncológico/inmunológicas, no hay mucha evidencia de comorbilidades en pacientes jóvenes.

EI INER es centro de referencia no pediátrico para atención de pacientes COVID-19 en forma moderada y grave. En nuestro país el problema de obesidad afecta a todos los grupos de edad. En México, 70\% de los mexicanos padece sobrepeso y casi una tercera parte sufre de obesidad, además, esta enfermedad se asocia principalmente con la diabetes y enfermedades cardiovasculares, pero también con trastornos óseos y musculares y algunos tipos de cáncer. En la actualidad, sabemos que estas comorbilidades son las que han demostrado un curso más tórpido
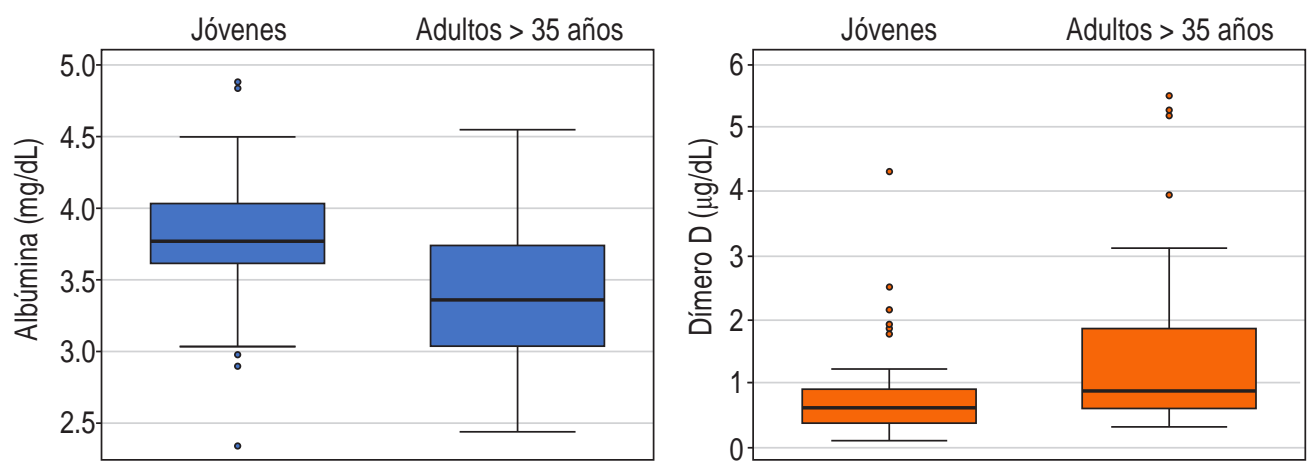

Figura 2:

Comparación de valores de albúmina y dímero $D$ en jóvenes y adultos $\geq 35$ años. Gráficos box plots. Diferencia significativa en ambos grupos $p<0.05$. 
de la enfermedad COVID-19. Consideramos la influencia de la epidemiología local para los hallazgos en nuestro estudio respecto a las comorbilidades reportadas, ya que encontramos que las comorbilidades más frecuentes fueron obesidad, sobrepeso, diabetes tipo 2, hipertensión arterial sistémica y asma.

En términos de gravedad de COVID-19 y fallecimiento en México, las principales patologías que están contribuyendo al desenlace fatal, según la Secretaría de Salud en su informe actualizado a principios de agosto 2020, fueron de manera general: hipertensión arterial sistémica 43\%; diabetes tipo 2 38\%; obesidad 25\%; insuficiencia renal crónica 7\%; enfermedad pulmonar obstructiva crónica y enfermedad cardiovascular 5\% de todas las muertes registradas. En contraste, $27 \%$ no presentó comorbilidades. ${ }^{17}$

Respecto a la edad como único factor predictor de mal pronóstico, en nuestro estudio los jóvenes con enfermedad COVID-19 que ameritaron ingreso presentaron comorbilidades como obesidad, sobrepeso y trastornos metabólicos (DM2); sin embargo, es de interés clínico que reportaron menores grados de taquipnea, gravedad de COVID-19 y letalidad por infección por SARS-CoV-2 al ser comparados con adultos (15 versus 45\% $\mathrm{p}<0.05$ ).

Otros estudios realizados en población mexicana también han reportado de manera similar la carga de comorbilidades asociadas a mal pronóstico en esta enfermedad. Hernández-Galdamez et al. reportaron que hasta $47.40 \%$ de los pacientes con diagnóstico de COVID-19 notificaron alguna comorbilidad, siendo la hipertensión la más frecuente $(20.12 \%) .^{18}$ Respecto a la edad, Ortiz Brizuela et al. reportaron que, en comparación con los pacientes ambulatorios, los pacientes hospitalizados eran de mayor edad, además de que tenían comorbilidades. ${ }^{19}$ Asimismo, Parra-Bracamonte et al. reportaron que la edad, el sexo y las comorbilidades más frecuentes, diabetes, obesidad e hipertensión, se asociaron significativamente con el riesgo de muerte por COVID-19 $(p<0.0001){ }^{20}$

Tabla 3: Asociación de jóvenes y adultos $\geq 35$ años con muerte ( $\mathrm{N}=102)$.

\begin{tabular}{|l|c|c|}
\hline Características & OR (IC 95\%) & p \\
\hline Adultos jóvenes $<35$ años & $0.22(0.088-0.57)$ & 0.002 \\
\hline Adultos $\geq 35$ años & $4.4(1.75-11.04)$ & 0.002 \\
\hline Relación neutrófilos/linfocitos $>3.3$ & $8.03(1.01-63)^{\star}$ & 0.049 \\
\hline Relación plaquetas/linfocitos $>180$ & $3.6(1.19-11.18)$ & 0.028 \\
\hline Dímero D & $1.10(1.01-1.21)$ & 0.026 \\
\hline Albúmina & $0.18(0.066-0.49)$ & 0.001 \\
\hline
\end{tabular}

$\mathrm{OR}=$ odds ratio, $\mathrm{IC}=$ intervalo de confianza.

* OR calculado por modelo de regresión logística con desenlace de muerte versus vivo.
En pacientes con enfermedad COVID-19 de espectro grave-crítico, los estudios en población mexicana documentan que, en comparación con los sobrevivientes, los no sobrevivientes eran mayores y tenían más probabilidades de tener diabetes, hipertensión u otras afecciones, dejando en claro el papel de las enfermedades crónicas y metabólicas en el impacto pronóstico de los pacientes. ${ }^{21}$

El papel de los biomarcadores inflamatorios en enfermedad COVID-19 es más claro en el grupo de formas graves y críticas, siendo algunos de ellos fuertes predictores independientes de deterioro respiratorio o de mortalidad.

Ai-Ping Yang et al. en su estudio de biomarcadores inflamatorios encontraron que el umbral del índice neutrófilo/ linfocito $(\mathrm{RNL})$ arriba de 3.3 y relación plaquetas/linfocito (RPL) arriba de 180 mostraba una posibilidad pronóstica superior de los síntomas clínicos. En nuestro estudio observamos que la $R N L \geq 3.3$ y $R P L \geq 180$ se encontraron como posibles biomarcadores predictores de deterioro por COVID-19 con un OR de 8.03 y 3.6, respectivamente. ${ }^{22}$

Otros biomarcadores que se han investigado al ingreso de pacientes enfermos de COVID-19 para predecir deterioro por fallo respiratorio o muerte son albumina, DHL, pro-BNP; sin embargo, la evidencia no ha sido concluyente. Por el contrario, sabemos que el papel desempeñado por el D-dímero se relaciona con el alto estado procoagulable en COVID-19, como se muestra por la disminución notable en mortalidad cuando esos con altos niveles del D-dímero se tratan con la anticoagulación. Zhang et al. en 2020 demostraron que el dímero $\mathrm{D}$ podría ser un marcador temprano y útil para mejorar el tratamiento de los pacientes con COVID-19, reportando que un dímero $\mathrm{D}$ al ingreso mayor de $2.0 \mu \mathrm{g} / \mathrm{mL}$ (aumento de cuatro veces) podría predecir eficazmente la mortalidad hospitalaria en pacientes con COVID-19. ${ }^{23}$

\section{CONCLUSIÓN}

Los adultos jóvenes con COVID-19 que ameritan hospitalización tienen un alto porcentaje de obesidad similar a los adultos mayores de 35 años. Sin embargo, destacan en la diferencia en biomarcadores con mayor cantidad de albumina inicial, y niveles más bajos de dímero $\mathrm{D}$ con cuadros comúnmente leves-moderados al ingreso y menos letales comparados con los enfermos adultos hospitalizados.

\section{REFERENCIAS}

1. World Health Organization. Coronavirus disease 2019 (COVID-19) situation report-97. Geneva: World Health Organization; 2020. Tomado de: https://www.who.int/docs/default-source/coronaviruse/situationreports/20200426-sitrep-97-covid-19.pdf?sfvrsn=d1c3e800_6

2. Wang L, He W, Yu X, Hu D, Bao M, Liu H, et al. Coronavirus disease 2019 in elderly patients: characteristics and prognostic factors based 
on 4-week follow-up. J Infect. 2020;80(6):639-645. Available in: https:// doi.org/10.1016/j.jinf.2020.03.019

3. Masters PS. The molecular biology of coronaviruses. Adv Virus Res. 2006;66:193-292. Available in: https://doi.org/10.1016/s00653527(06)66005-3

4. Kang S, Peng W, Zhu Y, Lu S, Zhou M, Lin W, et al. Recent progress in understanding 2019 novel coronavirus (SARS-CoV-2) associated with human respiratory disease: detection, mechanisms and treatment. Int J Antimicrob Agents. 2020;55(5):105950. doi: 10.1016/j. ijantimicag.2020.105950.

5. Gorbalenya AE, Baker SC, Baric RS, de Groot RJ, Drosten C, Gulyaeva AA, et al.; Coronaviridae Study Group of the International Committee on Taxonomy of Viruses. The species severe acute respiratory syndrome-related coronavirus: classifying 2019-nCoV and naming it SARS-CoV-2. Nat Microbiol. 2020;5(4):536-544. Available in: https://doi.org/10.1038/s41564-020-0695-z

6. Zhou P, Yang XL, Wang XG, Hu B, Zhang L, Zhang W, et al. A pneumonia outbreak associated with a new coronavirus of probable bat origin. Nature. 2020;579(7798):270-273. Available in: https://doi. org/10.1038/s41586-020-2012-7

7. Report S. Novel coronavirus (2019-nCoV) situation report-22. Geneve: World Health Organization; 2020. [Google Scholar]

8. World Health Organization. Modes of transmission of virus causing COVID-19: implications for IPC precaution recommendations. Geneva: World Health Organization; 2020. pp. 10-12. [Google Scholar]

9. Xu Y, Li X, Zhu B, Liang H, Fang C, Gong Y, et al. Characteristics of pediatric SARS-CoV-2 infection and potential evidence for persistent fecal viral shedding. Nat Med. 2020;26(4):502-505. Available in: https://doi.org/10.1038/s41591-020-0817-4

10. Hindson J. COVID-19: faecal-oral transmission? Nat Rev Gastroenterol Hepatol. 2020;17(5):259. Available in: https://doi. org/10.1038/s41575-020-0295-7

11. Chen N, Zhou M, Dong X, Qu J, Gong F, Han Y, et al. Epidemiological and clinical characteristics of 99 cases of 2019 novel coronavirus pneumonia in Wuhan, China: a descriptive study. Lancet. 2020;395(10223):507-513. Available in: https://doi.org/10.1016/s0140-6736(20)30211-7

12. Zhu N, Zhang D, Wang W, Li X, Yang B, Song J, et al. A novel coronavirus from patients with pneumonia in China, 2019. N Engl J Med. 2020;382(8):727-733. Available in: https://doi.org/10.1056/ nejmoa2001017

13. Zhou F, Yu T, Du R, Fan G, Liu Y, Xiang J, et al. Clinical course and risk factors for mortality of adult inpatients with COVID-19 in Wuhan, China: a retrospective cohort study. Lancet. 2020;395(10229):10541062. Available in: https://doi.org/10.1016/s0140-6736(20)30566-3

14. Nishiura $H$, Kobayashi T, Miyama T, Suzuki A, Jung SM, Hayashi $\mathrm{K}$, et al. Estimation of the asymptomatic ratio of novel coronavirus infections (COVID-19). Int J Infect Dis. 2020;94:154-155. Available in: https://doi.org/10.1016/j.ijid.2020.03.020

15. Libertini $G$, Corbi G, Cellurale M, Ferrara N. Age-related dysfunctions: evidence and relationship with some risk factors and protective drugs. Biochemistry (Mosc). 2019;84(12):1442-1450. Available in: https://doi. org/10.1134/s0006297919120034

16. Swann OV, Holden KA, Turtle L, Pollock L, Fairfield CJ, Drake TM, et al. Clinical characteristics of children and young people admitted to hospital with COVID-19 in United Kingdom: prospective multicentre observational cohort study. BMJ. 2020;370:m3249. Available in: https://doi.org/10.1136/bmj.m3249

17. COVID-19 México. [Fecha de consulta: 10 de septiembre de 2020] Disponible en: https://www.debate.com.mx/salud/ Covid-19-Mexico--23-de-julio-Suman-41908-muertes-y-370712contagios-20200723-0236.html

18. Hernández-Galdamez DR, González-Block MA, Romo-Dueñas DK, Lima-Morales R, Hernández-Vicente IA, Lumbreras-Guzmán M, et al. Increased risk of hospitalization and death in patients with COVID-19 and pre-existing noncommunicable diseases and modifiable risk factors in Mexico. Arch Med Res. 2020;51(7):683-689. Available in: https://doi.org/10.1016/j.arcmed.2020.07.003

19. Ortiz-Brizuela E, Villanueva-Reza M, González-Lara MF, TamezTorres KM, Román-Montes CM, Díaz-Mejía BA, et al. Clinical and epidemiological characteristics of patients diagnosed with covid-19 in a tertiary care center in Mexico City: a prospective cohort study. Rev Invest Clin. 2020;72(3):165-177. Available in: https://doi.org/10.24875/ ric. 20000211

20. Parra-Bracamonte GM, Lopez-Villalobos N, Parra-Bracamonte FE. Clinical characteristics and risk factors for mortality of patients with COVID-19 in a large data set from Mexico. Ann Epidemiol. 2020;52:9398.e2. Available in: https://doi.org/10.1016/j.annepidem.2020.08.005

21. Ñamendys-Silva SA, Alvarado-Ávila PE, Domínguez-Cherit G, Rivero-sigarroa E, Sánchez-Hurtado LA, Gutiérrez-Villaseñor A, et al. Outcomes of patients with COVID-19 in the intensive care unit in Mexico: A multicenter observational study. Heart Lung. 2021;50(1):2832. Available in: https://doi.org/10.1016/j.hrtlng.2020.10.013

22. Yang AP, Liu JP, Tao WQ, Li HM. The diagnostic and predictive role of NLR, d-NLR and PLR in COVID-19 patients. Int Immunopharmacol. 2020;84:106504. Available in: https://doi.org/10.1016/j. intimp.2020.106504

23. Zhang L, Yan X, Fan Q, Liu H, Liu X, Liu Z, et al. D-dimer levels on admission to predict in-hospital mortality in patients with COVID-19. J Thromb Haemost. 2020;18(6):1324-1329. Available in: https://doi. org/10.1111/jth.14859

Conflicto de intereses: Los autores declaran no tener conflicto de intereses. 